\title{
Aplicação da lógica fuzzy no estudo do risco de ataque cardíaco
}

\author{
Naara Karolyne Morais Pereira \\ Instituto Federal de Educação, Ciências e Tecnologia - Goiás \\ 76400-000, Campus Uruaçu, Uruaçu, GO \\ E-mail: naarakarolyne87@yahoo.com.br \\ Graciele Paraguaia Silveira \\ Universidade Federal de Goiás - Regional Jataí \\ 75801-615, Coordenação de Matemática, Jataí, GO \\ E-mail: gracimat@gmail.com
}

\section{$\underline{\text { RESUMO }}$}

Segundo a Organização Mundial de Saúde (OMS), as doenças cardiovasculares, como infartos e acidentes vasculares cerebrais (AVC), ainda constituem a primeira causa de mortes no mundo, com 17 milhões de mortos em 2011.

O objetivo do modelo proposto neste trabalho é predizer o risco de ataque cardíaco ${ }^{1}$, tendo em vista que doenças cardiovasculares há tempos também são a principal causa de mortes no Brasil.

De acordo com Varella (2011) [4], para se avaliar o risco de ataque cardíaco, deve-se levar em conta os seguintes fatores: limites para os valores do colesterol, número de fatores de risco apresentados pelo indivíduo e nível da proteína C-reativa.

Os principais fatores de risco definidos pelo National Cholesterol Education Program são:

- Idade: homens a partir dos 45 anos e mulheres depois da menopausa;

- Fumo;

- Hipertensão: mesmo quando tratada;

- Diabetes;

- História de doença cardiovascular em parentes de primeiro grau: doenças instaladas em parentes masculinos antes dos 55 anos de idade ou em parentes femininos com menos de 65 anos.

- Níveis de HDL abaixo de $35 \mathrm{mg}$ por dl.

Para diminuir o risco de ataque cardíaco e manter os níveis do colesterol adequados é preciso controlar o peso corpóreo e praticar atividade física, mas quando estas medidas falham, os médicos aconselham reduzir a quantidade de gordura na alimentação e, eventualmente, prescrevem medicamento.

A relação entre os altos níveis de LDL, o "mau" colesterol, e uma proteína chamada C-reativa no sangue fornecem as explicações mais convincentes sobre as principais causas para a instalação de placas de gordura nas artérias que irrigam o coração [4].

Os ataques cardíacos são provenientes de coronárias entupidas por placas de colesterol; quanto mais gordura no sangue, mais rápida a formação da placa e maior a probabilidade de obstrução.

No final de 1960, passou-se a acreditar que determinar o valor das concentrações no sangue de HDL, "bom" colesterol, e LDL, "mau" colesterol se podia determinar o prognóstico, pois segundo os médicos quanto mais alto o LDL, maior seria o risco de infarto do miocárdio (ataque cardíaco), derrame cerebral e complicações vasculares.

\footnotetext{
${ }^{1}$ Este trabalho foi desenvolvido durante o curso de Especialização da primeira autora.
} 
No entanto, haviam evidentes contradições:

1) Há pessoas que nunca enfartam apesar de apresentarem extensas placas, que obstruem significativamente a luz das coronárias. Outras que possuem placas insignificantes, com pequeno grau de obstrução, podem sofrer infartos extensos;

2) Os níveis altos de colesterol explicam apenas $50 \%$ dos episódios de infarto, a outra metade dos eventos ocorre em pessoas com colesterol normal;

3) As estatinas, drogas utilizadas para reduzir as concentrações de colesterol no sangue, administradas a pessoas com LDL elevado, diminuem a probabilidade de ataques cardíacos e derrames cerebrais. Mesmo as pessoas com níveis normais de LDL, podem se beneficiar do uso desses medicamentos.

A partir dos anos 90, pesquisas apontaram que o infarto ocorre quando substâncias resultantes das reações inflamatórias que ocorrem no interior da placa, digerem a cápsula protetora e provocam a formação de coágulos. Uma das substâncias envolvidas nesse processo é a proteína C-reativa, cujo nível está diretamente associado com as ocorrências de ataques cardíacos e sua alteração se dá também na presença dos demais fatores de risco.

Diante disso, optamos por construir um sistema baseado em regras fuzzy [1, 3], considerando como variáveis de entrada: Número de fatores de risco, variando de zero à sete e portador de doença cardiovascular; Níveis de LDL, classificados como baixo - menores que 130, médio - níveis entre 130 e 159 e alto - níveis maiores que 160 [2], e Proteína C-reativa, cujos termos linguísticos atribuídos foram possivelmente baixo - entre 0 e 0.3 e possivelmente alto - entre 0.3 e 0.5 .

A variável de saída risco de ataque cardíaco foi classificada como baixo, médio e alto. A base de regras fuzzy foi elaborada tendo como referência as informações obtidas na literatura. O método de inferência foi o de Mamdani e a defuzzificação realizada via Método do Centro de Gravidade.

Os resultados das simulações mostram o grau de pertinência de um paciente com determinado quadro clínico, aos conjuntos fuzzy associados ao risco de ataque cardíaco. Por exemplo, um paciente que apresenta um número de fatores de risco igual a 4, LDL igual a $100 \mathrm{mg} / \mathrm{dl}$ e proteína C-reativa igual a 0,5 , após a defuzzificação o risco de ataque cardíaco será de 0,81 , cuja a pertinência no conjunto risco alto é 1 .

Em outra situação, se um paciente é portador de doença cardiovascular, possui LDL igual 50 e proteína C-reativa igual 0,3 , então o risco de ataque cardíaco será de 0,5 , cuja pertinência é 1 para médio risco de ataque cardíaco.

Em trabalhos futuros, pretende-se realizar um estudo mais rigoroso dos parâmetros envolvidos no problema e validar os resultados obtidos pelo modelo, por meio da avaliação de especialistas e da pesquisa de resultados já comprovados, para comparações.

Palavras-chave: Lógica Fuzzy, Risco de Ataque Cardíaco, Nivel de LDL, Proteína C-reativa.

\section{Referências}

[1] L. C. Barros; R. C. Bassanezi, Tópicos de Lógica Fuzzy e Biomatemática. IMECC-UNICAMP, Campinas, 2012.

[2] M. C. Bertolami, Colesterol Alto. Artigos Médicos. Disponível em: http://emedix.uol.com.br/doe/car006_1h_colesterolalto.php. Acesso em: 05 Mar. 2014.

[3] R. S. M. Jafelice; L. C. Barros; R. C. Bassanezi, Usando a Teoria dos Conjuntos Fuzzy na Modelagem de Fenômenos Biológicos. In: Simpósio de Aplicações em Lógica Fuzzy, Sorocaba, SP, 2008 .

[4] D. Varella, Proteína C-reativa e acidentes cardiovasculares. Disponível em: http://drauziovarella.com.br/doencas-e-sintomas/proteina-c-reativa/. Acesso em: 05 Mar. 2014. 